\title{
A NOVEL CROSS-TALK BETWEEN PERIVASCULAR ADIPOSE TISSUE AND THE ARTERIAL WALL CONTROLS REDOX STATE IN HUMAN ATHEROSCLEROSIS
}

M Margaritis, ${ }^{1}$ A Antonopoulos, ${ }^{1}$ P Coutinho, ${ }^{1}$ M Petrou, ${ }^{2}$ R DeSilva, ${ }^{2}$ R Sayeed, ${ }^{2}$ K Channon, ${ }^{1} \mathrm{C}$ Antoniades ${ }^{1}{ }^{1}$ University of Oxford; ${ }^{2} J o h n$ Radcliffe Hospital

doi:10.1136/heartjnl-2013-304019.269

Background Endothelial nitric oxide synthase (eNOS) plays a crucial role in maintenance of vascular homeostasis. However, loss of eNOS co-factor tetrahydrobiopterin (BH4) due to oxidative degradation leads to uncoupling of the enzyme, that is turned into a source of superoxide radicals $\left(\mathrm{O}_{2}^{-}\right)$instead of nitric oxide (NO). Adipose tissue has been identified as the source of hormone-like molecules termed adipokines, which can exert endocrine and paracrine effects on the vascular wall. However, little is known about the role of adipokines such as adiponectin (AdN) in the regulation of vascular redox signalling in the human arterial wall or the mechanisms regulating their synthesis in perivascular adipose tissue (PVAT).

Methods In Study 1, 677 patients undergoing coronary bypass surgery $(\mathrm{CABG})$ were recruited. Blood samples were obtained preoperatively and internal mammary artery (IMA) segments as well as PVAT surrounding them were harvested during surgery. Serum AdN was quantified ELISA, vascular $\mathrm{O}_{2}^{-}$was determined by lucigenin chemiluminescence (+/-LNAME to estimate eNOS coupling) and qRTPCR was performed to determine AdN gene expression in PVAT. In Study 2, IMA segments from 17 patients undergoing CABG were exposed to AdN $(10 \mu \mathrm{g} / \mathrm{ml}, 6 \mathrm{~h}) \pm$ wortmannin (W, a PI3K/Akt inhibitor) ex vivo to determine the effects of $\mathrm{AdN}$ on vascular redox state, 


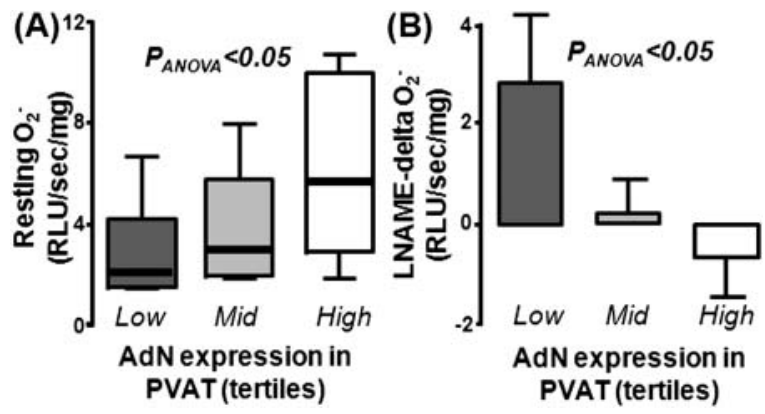

Figure 1

(C)

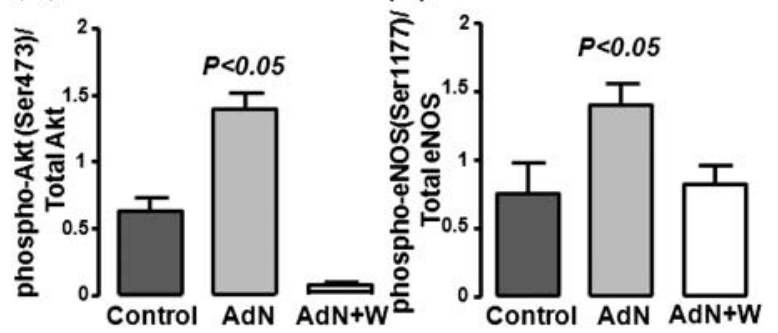

Figure 2

eNOS phosphorylation status and BH4 content. In addition, PVAT was exposed to 4-hydroxynonenal (4HNE, a peroxidation product released by the vascular wall in the presence of high vascular oxidative stress) ex vivo to determine its effects on AdN gene expression.

Results In Study 1, serum AdN was inversely related with resting $\mathrm{O}_{2}^{-} \quad(\mathrm{p}<0.01)$ and positively with the degree of eNOS coupling $(p<0.05)$ in the IMA. However, the expression of AdN gene in PVAT was positively related with resting O2- (A) and eNOS uncoupling $(B)$ in the same vessels. In Study $2, A d N$ reduced $\mathrm{O}_{2}^{-}(p<0.05)$ by restoring eNOS coupling $(p<0.05)$ in human arteries ex vivo. This was due to Akt-dependent eNOS phosphorylation at Ser1177 $(\mathrm{C} \& \mathrm{D})$ and an increase in vascular $\mathrm{BH} 4(\mathrm{p}<0.05)$. Vascular $\mathrm{O}_{2}^{-}$triggered the release of $4 \mathrm{HNE}$, while ex vivo exposure of PVAT to $4 \mathrm{HNE}$ up-regulated the expression of PPAR $\gamma(\mathrm{P}<0.05)$ and subsequently the expression of AdN gene $(\mathrm{P}<0.05)$.

Conclusions We describe a novel cross-talk between adipose tissue and the vascular wall in humans. Oxidative stress triggers the release of 4HNE from the vascular wall, which in turn up-regulates the expression of AdN in PVAT; then AdN exerts a paracrine effect on the vascular wall by activating eNOS and improving its BH4-mediated coupling. 